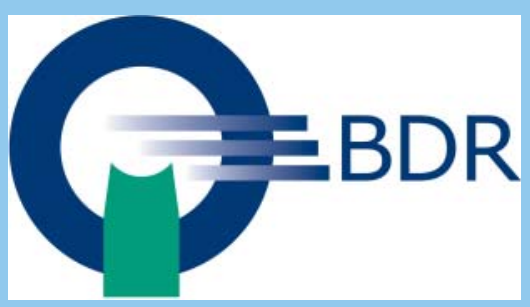

\title{
Strukturwandel in der Radiologie
}

\section{Erhalt der ärztlichen Freiberuflichkeit - Interessenwahrung angestellter Ärzte}

\section{Delegiertenversammlung 2018}

Die Delegiertenversammlung, das bedeutendste Gremium des BDR, tagte wie immer im Februar, diesmal am 17. 2. 2017, um Rechenschaft über das zurückliegende Jahr abzugeben, die Aufgaben und Ziele des BDR für das laufende Jahr zu diskutieren und festzulegen und in diesem Jahr auch den Vorstand neu zu wählen.

Dr. Detlef Wujciak, 1. Vorsitzender des BDR, fasste das zurückliegende Jahr 2017 für den BDR zusammen.

\section{Heuschrecken vs. Freiberufler}

Einer der Schwerpunkte war und ist die Zunahme der nichtärztlich geführten MVZ in der Radiologie und die damit verbundene Entfernung eines ethisch begründeten Handelns gegenüber den Patientlnnen. Hierzu beleuchtete RA Markus Henkel in seinem Vortrag mit dem Titel „Heuschrecken und Freiberufler in der Radiologie“ die juristischen Aspekte, die zur Beschränkung des Engagements externer Investoren genutzt werden könnten.

\section{Keine Regierung - keine Weiterent- wicklung der Gebührenordnungen?}

Zu dem existenziellen Thema Entwicklung der Gebührenordnungen - GOÄ wie auch EBM neu - gibt es derzeit nichts Neues aus Berlin. Das vorläufige Ergebnis der bereits seit Mai 2016 laufenden GOÄ neu-Gespräche zwischen BÄK, Berufsverbänden und PKV ist ein novelliertes Leistungsverzeichnis. Dies besteht aus 5589 Leistungspositionen, somit ca. 2700 mehr als bisher, unterteilt in 4196 Haupt- und 1393 Zuschlagsleistungen. Nun fehlen noch die Bewertungen. Zu zeitnahen Vorschlägen wird es wohl nicht kommen, da im aktuellen Koalitionsvertrag vereinbart wurde, eine wissenschaftliche Kommission zu etablieren, die bis Ende 2019 Vorschläge für ein „modernes Vergütungssystem“ für GKV und PKV erarbeiten soll. Bis dahin ruht die GOÄ neu dann wohl und wird 2020 oder 2021 wieder angegangen.

\section{Zeitnaher Zugang zu Innovationen für Patienten}

Ein so innovatives Fach wie die Radiologie leidet besonders unter den zeitlichen Längen, bis eine neue Methode durch das Procedere des Gemeinsamen Bundesausschusses für PatientInnen verfügbar wird. Prostata-MRT und Tomosynthese als neue Methoden werden weiter stringent von uns verfolgt.

\section{Dachverband der ärztlichen Diag- nostikfächer (DVÄD)}

Die fünf Berufsverbände (Pathologie, Labormedizin, BÄMI, Nuklearmedizin und Radiologie) eint, dass sie medizinisch hoch innovativ sind, sektoren- und fachübergreifend mit höchsten Qualitätsmaßstäben arbeiten, aber trotzdem nachgewiesen unterfinanziert sind. Der von uns mitgegründete DVÄD (www.dväd.de) ist deshalb seit vergangenem Jahr dabei, die besondere Stellung der Diagnostikfächer gegenüber Politik und Selbstverwaltung darzustellen.

\section{Kontrastmittel in Europa}

Zu diesem komplexen Thema weisen wir besonders auf den Beitrag von Prof. Hamm zur Entscheidung der Europäischen Kommission zu gadoliniumhaltigen Kontrastmitteln (GBCAs) in der Januar-Ausgabe des RADIOLOGEN (S. 95) hin. Per Mail können Sie ihn anfordern unter presse@radiologenverband.de

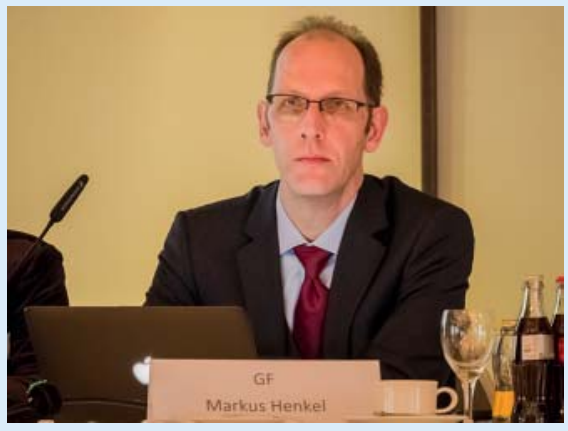

GF Henkel sprach über die HeuschreckenPlage in der Radiologie

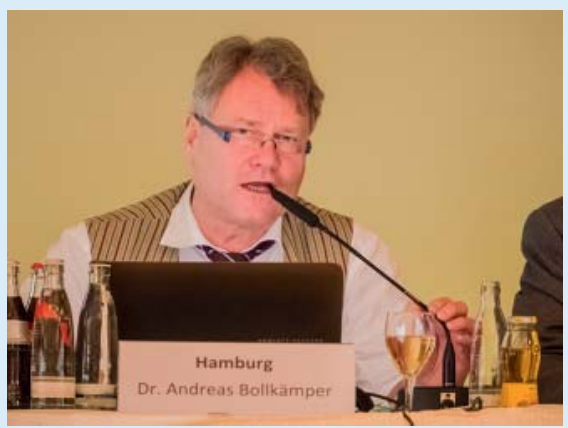

Kassenwart Dr. Bollkämper steht Rede und Antwort zum Finanzbericht

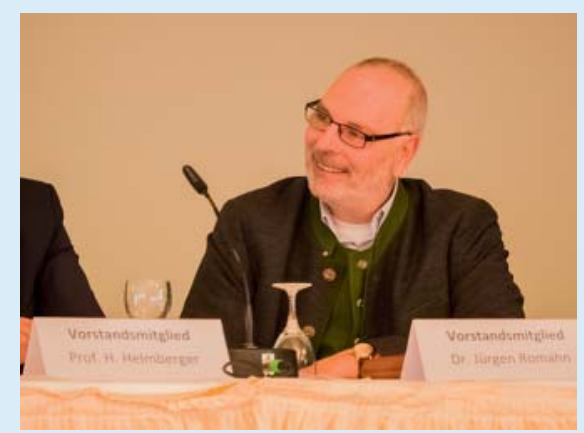

Ein harmonischer Abschied von Dr. Romahn 


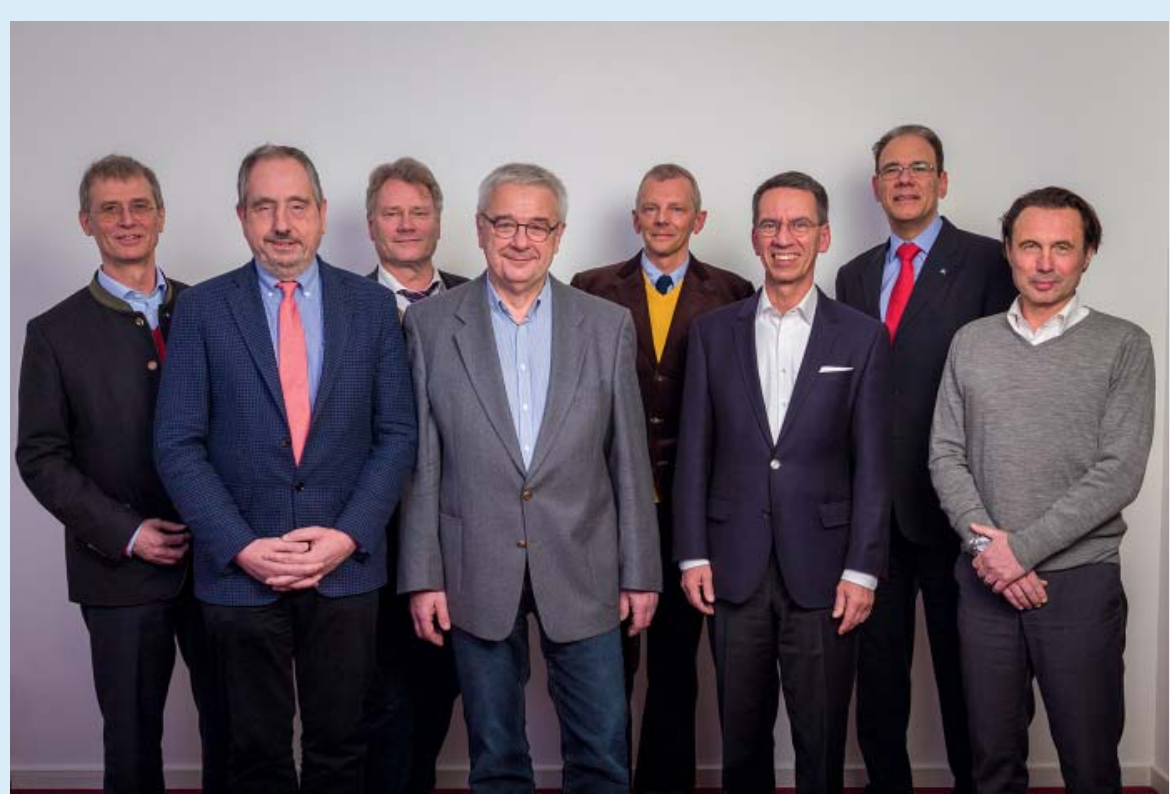

Der neu gewählte Vorstand: Dr. J. Köpke, Dr. W. Schaeben, Dr. A. Bollkämper, Dr. K. Hamm, Dr. D. Wujciak, Prof. B. Hamm, Prof. H. Helmberger, S. Schmidt

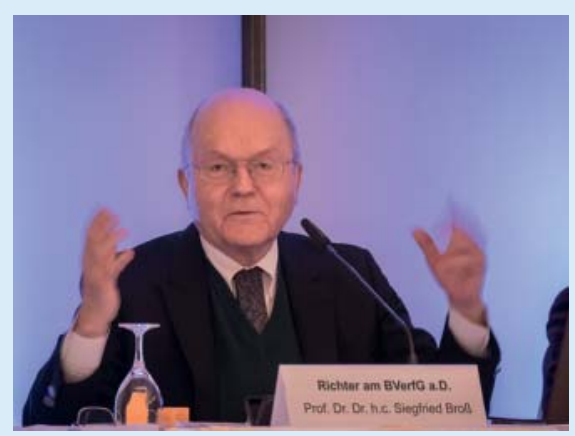

Prof. Broß referiert engagiert zum Thema Krankenfürsorge

\section{MTRA als Mangelberuf}

Die Nachwuchsgewinnung ist nicht nur auf der ärztlichen Seite problematisch, sondern auch im Bereich von Praxis-und Klinikpersonal evident. Die Problematik MTRA als Mangelberuf und mögliche Ansätze zur Lösung des Problems werden in diesem Jahr bearbeitet werden. Die Attraktivität des Berufs der MTA/MTRA werden wir, auch in Zusammenarbeit mit unseren Partnern des DVÄD, darstellen.
$\S 13$ Umbenennung der Vorstandspositionen

Hintergrund: alle anderen Berufsverbände haben gleichlautende Funktionsbezeichnungen und benennen Vorsitzende als Präsident bzw. stellvertretende Präsidenten.

\section{$\S 13$ Erweiterung des Vorstandes}

Hintergrund: die Aufgaben des Vorstandes nehmen in relevantem Umfang um neue Themenfelder und Aufgaben zu.

$\S 6$ Ende der Mitgliedschaft - Konkretisierung der Kündigungsfrist

Hintergrund: mit fixiertem Datum, zum 30.9., für den Austritt ergibt sich eine gröBere Planungssicherheit für Budgetplanung und Berechnung der Delegiertenzahlen.

\section{Vorstandswahlen 2018-22}

Die Wahl begann mit dem Dank an das ausscheidende Vorstandsmitglied Dr. Jürgen Romahn, der dem Vorstand zwei Legislaturperioden angehörte.

Alle Kandidaten wurden mit überwältigender Mehrheit gewählt.

\section{„Krankenfürsorge - ein Kultur- problem"}

Für das Jahr 2018 hat der BDR das Thema "Strukturwandel in der Radiologie“ als Arbeitsmotto gewählt. Hierzu stimmt der Vortrag des ehemaligen Verfassungsrichters Prof. Dr. Dr. h. c. Broß „Krankenfürsorge ein Kulturproblem“ bestens ein.

Der Vortrag wurde von ihm lebendig und eindrücklich vorgetragen und ebenso interessiert mit den Delegierten diskutiert. Unsere Mitglieder finden sein Redemanuskript auf unserer Webseite oder können per Mail angefordert werden (presse@radiologenverband.de).

Sönke Schmidt/Sabine Lingelbach 